\title{
QCD thermodynamics with dynamical overlap fermions
}

\author{
Szabolcs Borsányi ${ }^{a}$, Ydalia Delgado ${ }^{b}$, Stephan Dürr ${ }^{a, c}$, Zoltán Fodor $^{a, c, d}$, \\ Sándor D. Katz ${ }^{d}$, Stefan Krieg ${ }^{a, c}$, Thomas Lippert ${ }^{a, c}$, Dániel Nógrádi ${ }^{d}$, \\ Kálmán K. Szabó ${ }^{a, c, e}$ and Bálint C. Tóth ${ }^{* a}$ \\ ${ }^{a}$ Bergische Universität Wuppertal, D-42119 Wuppertal, Germany \\ ${ }^{b}$ Institut für Physik, Karl-Franzens-Universität, Graz, Austria \\ ${ }^{c} I A S$, Jülich Supercomputing Centre, Forschungszentrum Jülich, D-52425 Jülich, Germany \\ ${ }^{d}$ Institute for Theoretical Physics, Eötvös University, H-1117 Budapest, Hungary \\ ${ }^{e}$ Institute for Theoretical Physics, Universität Regensburg, D-93040 Regensburg, Germany \\ E-mail: tothbalinteszofi.elte.hu
}

\begin{abstract}
We extend our study of QCD thermodynamics using two flavors of dynamical overlap fermions to $N_{t}=10$ and $N_{t}=12$. We work in a fixed global topology setting with a quark mass corresponding to a pion mass of $350 \mathrm{MeV}$. We find a nice agreement with continuum results obtained with staggered fermions at the same pion mass.
\end{abstract}

31st International Symposium on Lattice Field Theory - LATTICE 2013

July 29 - August 3, 2013

Mainz, Germany

\footnotetext{
*Speaker.
} 


\section{Introduction}

At high temperatures the dominant degrees of freedom of strongly interacting matter change from hadrons to quarks and gluons. This transition can be studied using lattice gauge theory. There are various results using different fermion regularizations.

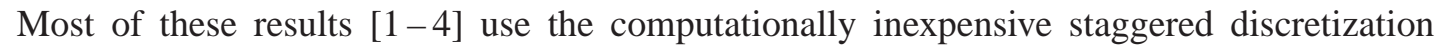
which also preserves a subset of the continuum chiral symmetry. Even though different staggered results seem to be consistent one should not forget that all these works use the fourth root trick to study $N_{f}=2+1$ flavors of quarks. There are still some questions in the literature about the correctness of this approach. Furthermore taste symmetry breaking may lead to large discretization errors when using small quark masses, especially at low temperatures.

There are also several results using Wilson fermions [5]-8]. Since Wilson fermions break chiral symmetry explicitly, one has to take very fine lattices to study chiral symmetry restoration at finite temperature. Due to the scattering of the low lying eigenvalues of the Wilson-Dirac operator, one needs large lattice volumes when going to small pion masses. There are also first results with twisted mass fermions [9].

It seems logical to use chiral fermions to study chiral properties at finite temperature. Even though chiral lattice fermions are computationally much more expensive than the other types of discretization, there are results in the literature using domain wall fermions [10] as well as first attempts with overlap fermions [11, 12]. While domain-wall fermions provide exact chiral symmetry only for an infinite extent of the fifth dimension, the overlap formulation [13, 14] has the advantage of exact symmetry on finite four dimensional lattices [15].

In this work we present results using two degenerate flavors of dynamical overlap fermions. We use four different lattice resolutions, corresponding to $N_{t}=6,8,10$ and 12 temporal extents. We determine the temperature dependence of the chiral condensate, the chiral susceptibility, the isospin susceptibility and the Polyakov loop. The results are compared to $N_{f}=2$ staggered data at the same pion mass.

\section{Overlap action and simulation details}

The possibility of using the Hybrid Monte Carlo algorithm (HMC) with overlap fermions was first discussed in Reference [16]. The overlap operator was implemented with a multi-shift inverter using the Zolotarev rational approximation [17]. It was observed that treating topology changes requires special care during the HMC trajectories. One has to track the lowest lying eigenvalues of the Wilson kernel of the overlap operator. This topic was studied in detail in References [18-20].

Reference [21] demonstrated that one can run simulations with a fixed topological charge in several different sectors, and that it is possible to determine their relative weight. However, even this approach requires a tracking of Wilson eigenvalues. In Reference [22] it was suggested that by adding an extra heavy Wilson fermion to the action which decouples in the continuum limit, one can disable topological sector changes and at the same time speed up the algorithm significantly. It was also claimed that in the thermodynamic limit physics is independent of the global topology and therefore this approach should give correct results. However, significant power-like finite volume corrections are expected [23, 24]. Here we follow the same approach: We add an extra Wilson 
fermion to suppress low lying eigenvalues of the Wilson kernel and disable tunneling between different topological sectors. As a further improvement we use smearing in the Wilson kernel. It was observed in [25, 26] that smearing significantly improves the properties of the overlap operator. Furthermore, since smearing decreases the eigenvalue density in the middle of the Wilson spectrum it results in a significant speedup of the algorithm [26].

In the gauge sector we use a tree-level Symanzik improved gauge action. The massive overlap operator can be written as

$$
D=\left(m_{0}-\frac{m}{2}\right)\left(1+\gamma_{5} \operatorname{sgn}\left(H_{W}\right)\right)+m,
$$

where $H_{W}=\gamma_{5} D_{W}$ is the Hermitian Wilson operator with a negative mass parameter $-m_{0}$ which is supposed to be in the range $-2<-m_{0}<0$ and $m$ is the mass of the overlap quark. For the Wilson kernel we use two steps of HEX smearing [27-29] with smearing parameters $\alpha_{1}=0.72, \alpha_{2}=0.60$ and $\alpha_{3}=0.44$. In order to set $m_{0}$ we evaluated the Wilson kernel on quenched configurations with the targeted lattice spacings and smearing parameters of this work and located the point which is in the middle between the physical modes and the first doublers. This resulted in $m_{0}=1.3$. The simulations are performed with $N_{f}=2$ flavors.

As suggested in [22] we add two irrelevant terms to the action to suppress low eigenvalues of $H_{W}$ and fix topology, so that the complete action takes the form

$$
S_{E}=\sum_{x}\left\{\bar{\psi}_{E}(x) D_{W}\left(-m_{0}\right) \psi_{E}(x)+\phi \dagger(x)\left[D_{W}\left(-m_{0}\right)+\mathrm{i} m_{B} \gamma_{5} \tau_{3}\right] \phi(x)\right\} .
$$

The first term is the action of two flavors of extra fermions with negative mass $-m_{0}$. The second term, including a two component bosonic field, is included to control the effect of the extra fermions. The eigenvalues of $H_{W}$ below $m_{B}$ are most strongly suppressed. Since both $m_{0}$ and $m_{B}$ are fixed in lattice units they correspond to infinitely large masses in the continuum limit and both these terms decouple. For the bosonic mass we use $m_{B}=0.54$. Since our lattice action results in a fixed topology we aimed at simulations with zero topological charge.

We use a HMC algorithm with the Hasenbusch trick [30], with an Omelyan integrator [31] and with a Sexton-Weingarten multi-scale scheme for the different fields [32]. The latter ingredient turned out to be rather advantageous; the extra Wilson fermion has to be integrated with a much smaller stepsize than the much more expensive overlap fermion.

The first step of our analysis was to determine the line of constant physics (LCP) and the scale. The lattice sizes used for this purpose were $12^{3} \times 24$ for couplings between 3.6 and 3.9, $16^{3} \times 32$ for $\beta$ values 4.0 and 4.1 , and $32^{4}$ for $\beta$ values 4.2 and 4.3 , with initial guesses for the bare quark masses between 0.015 and 0.06 . We determined the $w_{0}$ scale [33] as well as the pion masses on all of these lattices. Since due to the chiral symmetry of overlap fermions $m_{\pi}^{2} \propto m$ and the scale $w_{0}$ is quite insensitive to the quark mass, it was possible to tune the quark masses to have a fixed value of $m_{\pi} \cdot w_{0}=0.312$ for each beta without further simulations. With the physical value of $w_{0}$ at the $N_{f}=2+1$ flavor physical point, $w_{0}=0.1755 \mathrm{fm}$, this corresponds to $m_{\pi}=350 \mathrm{MeV}$. The lattice spacing as a function of $\beta$ and the LCP are shown in Figure 1. In one of our runs we had a (large) nonzero topological charge, $Q=20$. One can see from the figure that both the scale and the LCP have still a significant dependence on topology for our volumes. Accordingly, this $Q=20$ run is left out in the final analysis. 

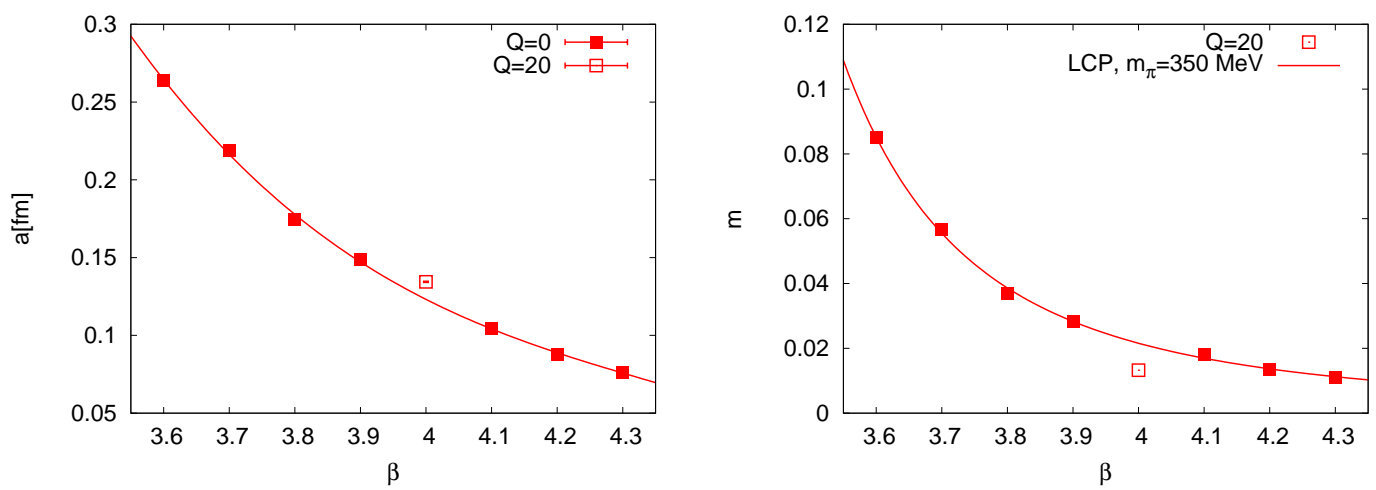

Figure 1: Left: The lattice spacing as a function of the coupling. The open box shows a run with a topological charge of $Q=20$ (not included in the fit). Right: The tuned bare quark mass $m$ as a function of $\beta$.

For the finite temperature calculations the aspect ratio was set to $N_{s} / N_{t}=2$, that is, the spatial extent was twice the size of the temporal extent. As a consequence $m_{\pi} \cdot L$ lies between 3.5 and 5 for all simulations in the transition regime.

\section{Results}

The first quantity we study is the chiral condensate, $\bar{\psi} \psi=(T / V) \partial / \partial m \log Z$. This can be renormalized using the zero temperature condensate $\bar{\psi} \psi_{0}$ (this observable was studied in [34])

$$
m_{R} \bar{\psi} \psi_{R} / m_{\pi}^{4}=m\left(\bar{\psi} \psi-\bar{\psi} \psi_{0}\right) / m_{\pi}^{4}
$$

and the renormalized condensate is plotted in the first panel of Figure 2 together with our staggered continuum estimate. One observes a broad cross-over, similar to the staggered results at physical quark masses [35].

We have also determined the chiral susceptibility

$$
\chi_{\bar{\psi} \psi}=(T / V) \partial^{2} / \partial m^{2} \log Z
$$

but at the present level of statistics the necessary renormalization step results in large errors. Therefore we only show the bare susceptibilities in the second panel of Figure 2.

The next quantity we study is the Polyakov loop. The bare Polyakov loop has a multiplicative divergence of the form $\exp \left[F_{0}(\beta) / T\right]$ where the divergent term $F_{0}$ can be determined up to a constant [36]. Different constants correspond to different renormalization schemes. We determine $F_{0}$ entirely from finite temperature simulations in the following way. We perform runs with nine different $N_{t}$ values, $N_{t}=4,5,6,7,8,9,10,11,12$; the lattice extents are $16^{3} \times N_{t}$. We choose a fixed physical temperature such that these $N_{t}$ values span our $\beta$ range. This corresponds to a temperature of $208 \mathrm{MeV}$ (having $N_{t}=9$ at $\beta=4.1$ ). From these runs we can determine $F_{0}(\beta)=1 / N_{t} \cdot \log L$ at nine $\beta$ values. This can then be extended by interpolation to all of our couplings. This renormalization scheme corresponds to the condition $L_{R}(T=208 \mathrm{MeV})=1$. The renormalized Polyakov loop is then given as

$$
L_{R}=L_{0} e^{-N_{t} \cdot F_{0}(\beta)},
$$



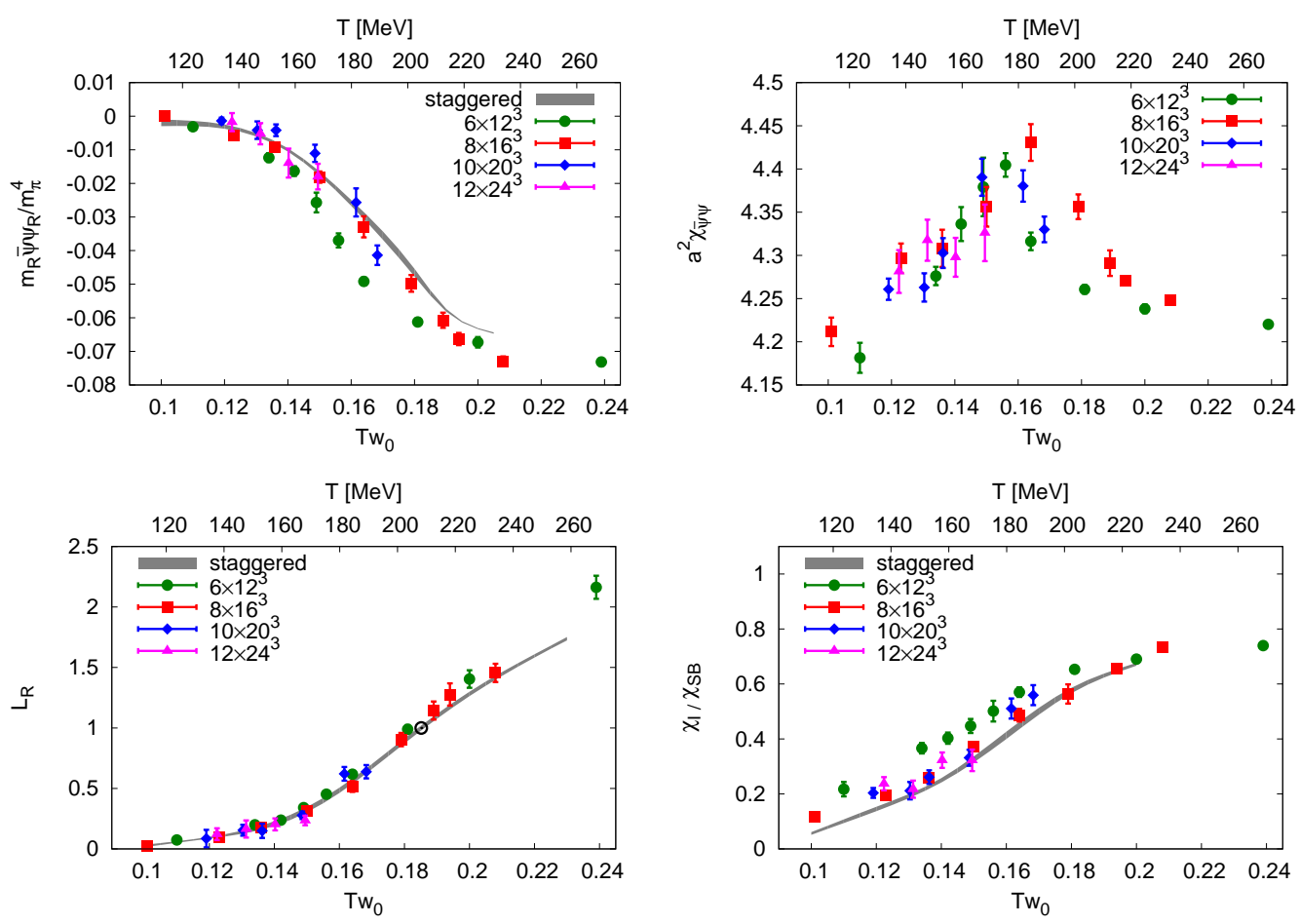

Figure 2: The renormalized chiral condensate (top left panel), bare chiral susceptibility (top right panel), renormalized Polyakov loop (bottom left panel) and the isospin susceptibility normalized by the respective Stefan-Boltzmann limits (bottom right panel) as a function of the temperature. The physical temperature scale (at the top boundary) is for illustration only and it is based on $w_{0}=0.1755 \mathrm{fm}$ [33]. The gray band indicates our staggered continuum estimate based on $N_{t}=6,8,10$ simulations.

where $L_{0}$ is the bare Polyakov loop. The result is shown in the third panel of Figure \&, where our renormalization condition $L_{R}(T=208 \mathrm{MeV})=1$ is represented by a black circle. We can see almost no lattice spacing dependence and an excellent agreement with the staggered results.

Our final observable is the isospin susceptibility,

$$
\chi_{I}=(T / V) \partial^{2} /\left.\partial \mu_{I}^{2} \log Z\right|_{\mu_{I}=0},
$$

where $\mu_{I}$ is the isospin chemical potential, i.e. the quark chemical potentials are $\mu_{u, d}= \pm \mu_{I} / 2$. Obtaining results at non-vanishing chemical potentials is very CPU demanding (see e.g. [37- 39]). Even though a detailed analysis to $\mu>0$ is beyond the scope of this work, including the chemical potential on the level of eq. (3.4) is quite interesting. The reason is that there is an ongoing discussion in the literature about the proper inclusion of the chemical potential in the overlap operator [40-42]. We follow Reference [40] and define the chemical potential as a fourth, imaginary component of the temporal gauge field and use the analytic continuation of the sign function $\operatorname{sgn}(z)=\operatorname{sgn} \operatorname{Re}(z)$. The second derivative can be calculated using the formulas of [12]. As a tree level improvement we normalize all susceptibilities with the corresponding Stefan-Boltzmann values. Our results for the isospin susceptibility are shown in the last panel of Figure 2 .

\section{Summary}

We presented results for the temperature dependence of several observables using two degen- 
erate flavors of dynamical overlap fermions with a pion mass of $m_{\pi}=350 \mathrm{MeV}$. We have used four different lattice spacings, corresponding to $N_{t}=6,8,10$ and 12. The errorbars on the finer lattices are still large, but at the current level of statistical precision we found no contradiction with the staggered results.

\section{Acknowledgments}

Computations were carried out on GPU [43] clusters at the Universities of Wuppertal and Budapest as well as on supercomputers in Forschungszentrum Juelich. This work is supported in part by the Deutsche Forschungsgemeinschaft grant SFB/TRR55, by OTKA under grant OTKANF-104034 and by the EU (FP7/2007-2013)/ERC No. 208740.

\section{References}

[1] Y. Aoki, S. Borsanyi, S. Durr, Z. Fodor, S. D. Katz, S. Krieg and K. K. Szabo, JHEP 0906 (2009) 088 [arXiv:0903.4155 [hep-lat]].

[2] S. Borsanyi et al. [Wuppertal-Budapest Collaboration], JHEP 1009 (2010) 073 [arXiv:1005.3508 [hep-lat]].

[3] A. Bazavov, H. -T. Ding, P. Hegde, O. Kaczmarek, F. Karsch, E. Laermann, Y. Maezawa and S. Mukherjee et al., Phys. Rev. Lett. 111, 082301 (2013) [Phys. Rev. Lett. 111 (2013) 082301] [arXiv:1304.7220 [hep-lat]].

[4] S. Borsanyi, G. Endrodi, Z. Fodor, A. Jakovac, S. D. Katz, S. Krieg, C. Ratti and K. K. Szabo, JHEP 1011 (2010) 077 [arXiv:1007.2580 [hep-lat]].

[5] V. G. Bornyakov, R. Horsley, S. M. Morozov, Y. Nakamura, M. I. Polikarpov, P. E. L. Rakow, G. Schierholz and T. Suzuki, Phys. Rev. D 82 (2010) 014504 [arXiv:0910.2392 [hep-lat]].

[6] S. Borsanyi, S. Durr, Z. Fodor, C. Hoelbling, S. D. Katz, S. Krieg, D. Nogradi and K. K. Szabo et al., JHEP 1208 (2012) 126 [arXiv:1205.0440 [hep-lat]].

[7] T. Umeda et al. [WHOT-QCD Collaboration], Phys. Rev. D 85 (2012) 094508 [arXiv:1202.4719 [hep-lat]].

[8] B. B. Brandt, A. Francis, H. B. Meyer, O. Philipsen and H. Wittig, arXiv:1310.8326 [hep-lat].

[9] E. -M. Ilgenfritz, K. Jansen, M. P. Lombardo, M. Muller-Preussker, M. Petschlies, O. Philipsen and L. Zeidlewicz, Phys. Rev. D 80 (2009) 094502 [arXiv:0905.3112 [hep-lat]].

[10] M. Cheng, N. H. Christ, M. Li, R. D. Mawhinney, D. Renfrew, P. Hegde, F. Karsch and M. Lin et al., Phys. Rev. D 81 (2010) 054510 [arXiv:0911.3450 [hep-lat]].

[11] G. Cossu et al. [JLQCD Collaboration], PoS LATTICE 2010 (2010) 174 [arXiv:1011.0257 [hep-lat]].

[12] S. Borsanyi, Y. Delgado, S. Durr, Z. Fodor, S. D. Katz, S. Krieg, T. Lippert and D. Nogradi et al., Phys. Lett. B 713 (2012) 342 [arXiv:1204.4089 [hep-lat]].

[13] H. Neuberger, Phys. Lett. B 417 (1998) 141 [hep-lat/9707022].

[14] H. Neuberger, Phys. Lett. B 427 (1998) 353 [hep-lat/9801031].

[15] M. Luscher, Phys. Lett. B 428 (1998) 342 [hep-lat/9802011]. 
[16] Z. Fodor, S. D. Katz and K. K. Szabo, JHEP 0408 (2004) 003 [hep-lat/0311010].

[17] J. van den Eshof, A. Frommer, T. Lippert, K. Schilling and H. A. van der Vorst, Comput. Phys. Commun. 146 (2002) 203 [hep-lat/0202025].

[18] T. A. DeGrand and S. Schaefer, Phys. Rev. D 71 (2005) 034507 [hep-lat/0412005].

[19] N. Cundy, S. Krieg, G. Arnold, A. Frommer, T. .Lippert and K. Schilling, Comput. Phys. Commun. 180 (2009) 26 [hep-lat/0502007].

[20] N. Cundy, S. Krieg, T. Lippert and A. Schafer, Comput. Phys. Commun. 180 (2009) 201 [arXiv:0803.0294 [hep-lat]].

[21] G. I. Egri, Z. Fodor, S. D. Katz and K. K. Szabo, JHEP 0601 (2006) 049 [hep-lat/0510117].

[22] H. Fukaya et al. [JLQCD Collaboration], Phys. Rev. D 74 (2006) 094505 [hep-lat/0607020].

[23] R. Brower, S. Chandrasekharan, J. W. Negele and U. J. Wiese, Phys. Lett. B 560 (2003) 64 [hep-lat/0302005].

[24] S. Aoki, H. Fukaya, S. Hashimoto and T. Onogi, Phys. Rev. D 76 (2007) 054508 [arXiv:0707.0396 [hep-lat]].

[25] T. G. Kovacs, Phys. Rev. D 67 (2003) 094501 [hep-lat/0209125].

[26] S. Durr, C. Hoelbling and U. Wenger, JHEP 0509 (2005) 030 [hep-lat/0506027].

[27] S. Capitani, S. Durr and C. Hoelbling, JHEP 0611 (2006) 028 [hep-lat/0607006].

[28] S. Durr, Z. Fodor, C. Hoelbling, S. D. Katz, S. Krieg, T. Kurth, L. Lellouch and T. Lippert et al., Phys. Lett. B 701 (2011) 265 [arXiv:1011.2403 [hep-lat]].

[29] S. Durr, Z. Fodor, C. Hoelbling, S. D. Katz, S. Krieg, T. Kurth, L. Lellouch and T. Lippert et al., JHEP 1108 (2011) 148 [arXiv:1011.2711 [hep-lat]].

[30] M. Hasenbusch, Phys. Lett. B 519 (2001) 177 [hep-lat/0107019].

[31] T. Takaishi and P. de Forcrand, Phys. Rev. E 73 (2006) 036706 [hep-lat/0505020].

[32] J. C. Sexton and D. H. Weingarten, Nucl. Phys. B 380 (1992) 665.

[33] S. Borsanyi, S. Durr, Z. Fodor, C. Hoelbling, S. D. Katz, S. Krieg, T. Kurth and L. Lellouch et al., JHEP 1209 (2012) 010 [arXiv:1203.4469 [hep-lat]].

[34] G. Endrodi, Z. Fodor, S. D. Katz and K. K. Szabo, JHEP 1104 (2011) 001 [arXiv:1102.1356 [hep-lat]].

[35] Y. Aoki, G. Endrodi, Z. Fodor, S. D. Katz and K. K. Szabo, Nature 443 (2006) 675 [hep-lat/0611014].

[36] Y. Aoki, Z. Fodor, S. D. Katz and K. K. Szabo, Phys. Lett. B 643 (2006) 46 [hep-lat/0609068].

[37] Z. Fodor and S. D. Katz, Phys. Lett. B 534 (2002) 87 [hep-lat/0104001].

[38] Z. Fodor and S. D. Katz, JHEP 0404 (2004) 050 [hep-lat/0402006].

[39] S. Borsanyi, G. Endrodi, Z. Fodor, S. D. Katz, S. Krieg, C. Ratti and K. K. Szabo, JHEP 1208 (2012) 053 [arXiv:1204.6710 [hep-lat]].

[40] J. C. R. Bloch and T. Wettig, Phys. Rev. Lett. 97 (2006) 012003 [hep-lat/0604020].

[41] R. V. Gavai and S. Sharma, Phys. Rev. D 81 (2010) 034501 [arXiv:0906.5188 [hep-lat]].

[42] R. Narayanan and S. Sharma, JHEP 1110 (2011) 151 [arXiv:1108.2667 [hep-lat]].

[43] G. I. Egri, Z. Fodor, C. Hoelbling, S. D. Katz, D. Nogradi and K. K. Szabo, Comput. Phys. Commun. 177 (2007) 631 [hep-lat/0611022]. 